\title{
Does Home Quarantine Ameliorate Different Antiviral Immune Elements
}

\section{Majid MM*}

Department of Biology, College of Science, Mustansiriyah University, Iraq

*Corresponding author: Majid Mohammed Mahmood, Department of Biology, College of Science, Mustansiriyah University, Iraq, Email: majidmahmood93@yahoo.com

\section{Opinion}

Volume 4 Issue 1

Received Date: March 27, 2020

Published Date: April 07, 2020

DOI: $10.23880 / v i j-16000231$

\section{Drastic Drop in World's Air Pollution after Coronavirus Quarantine Definitely Improves the Immune System Capabilities}

\section{Air Pollution and its Effects on the Immune System}

The immune system consists of multiple types of immune cells that act together to generate (or fail to generate) immune responses perfectly.

A well-functioning immune system is vital for a healthy body, with which pathology could be abrogated.

In this article we try to review evidences of how home quarantine can reinforce different immune cells types such as inflammatory neutrophils, particle-clearing macrophages, dendritic cells that orchestrate adaptive immune response and lymphocytes that ratify immune responses.

Inadequate and imperfect immune responses underlie diverse pathologies including viral infections, therefore, the effects of ambient pollutants on the immune system are of great importance and Air pollutants stimulate proinflammatory immune responses across multiple classes of immune cells, It can dysregulate anti-viral immune responses, the clinical effects of air pollution, in particular the known association between elevated ambient pollution, and exacerbations of asthma and chronic obstructive pulmonary disease (COPD). Further to this, inhaled air pollution deposits on the respiratory mucosa.

\section{Home Quarantine Mitigates Stress we used to Face in Work Environment}

\section{Stress-Induced Immune Dysfunction}

Immune system communicates bidirectional with the central nervous and endocrine systems and how these interactions impact on health. Stressful events have mischievous impact on health. Stress and the negative emotions can be translated into physiological changes.

The communication between the central nervous system and the immune system occurs via a complex network of bidirectional signals linking the nervous, endocrine and immune systems. The field of psychoneuroimmunology (PNI) has provided new insights to help understand the pathophysiological processes that are linked to the immune system. Work in this field has established.

Psychological stress disrupts the functional interaction between the nervous and immune systems. Stress-induced immune dysregulation has been shown to be significant enough to result in health consequences, including reducing the immune response to vaccines, reactivating latent herpes viruses, such as Epstein-Barr virus (EBV), and enhancing the risk for more severe infectious disease. Chronic stress/ depression can increase the peripheral production of proinflammatory cytokines. High serum levels of IL-6 have been linked to risks for several conditions, such as, mental health complications.

This overview is also to emphasize the evidence that psychological comfort promotes immune function that positively impacts human health and improve disease resistance.

Home Quarantine gave us a Good Opportunity to get a Variety of Rich, and Supportive Foods that Boost our Immune System we used not to Concern with During our Previous Daily Life

Malnutrition is associated with a significant impairment 


\section{Virology \& Immunology Journal}

of cell-mediated immunity, phagocyte function, complement system, secretory IgA antibody concentrations, and cytokine production and many other elements implicated in antivirus immunity.

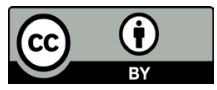

